\title{
Genes Induced by Panax Notoginseng in a Rodent Model of Ischemia-Reperfusion Injury
}

\author{
Lanqing Meng $\mathbb{D}^{1,2}$ Qing Huang ${ }^{1,},{ }^{2,3}$ Xuebin Li $\mathbb{D}^{2}$, Ping Liang, ${ }^{4}$ Yueyong Li $\mathbb{D}^{5}$

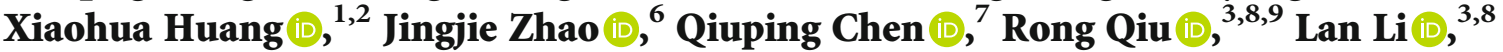 \\ Chongdong Jian $\mathbb{D}^{2}{ }^{2}$ Hongfei Yao, ${ }^{3}$ Jianmin Huang $\mathbb{D}^{2},{ }^{2}$ Xionglin Tang $\mathbb{D}^{2},{ }^{2}$ Zechen Wang ${ }^{(D)}, 8$ \\ Zhongheng Wei $\mathbb{D}^{5,8}$ Jun Wu ${ }^{10},{ }^{9}$ Liuzhi Wei $\mathbb{D}^{8,10}$ Qiuju Wei ${ }^{8},{ }^{8,10}$ Qianli Tang ${ }^{(D)}{ }^{8}$ \\ Lu Huang $\left(\mathbb{D},{ }^{3,8,9}\right.$ Jihua Wei $(\mathbb{D}){ }^{11}$ Dinggui Lu $\left(\mathbb{D},{ }^{11}\right.$ Qunqiang Luo $(\mathbb{D}),{ }^{11}$ Kegong Xie $\mathbb{D}^{1},{ }^{11}$ \\ Yang Ouyang $\mathbb{D}^{2}$ Jian Chen $\left(\mathbb{D},{ }^{3,11}\right.$ Genliang Li $\mathbb{D}^{12}$, Linxue Luo $\mathbb{D}^{13},{ }^{13}$ Linbo He $\mathbb{D}^{14}$ \\ Chenyi Zhuo $\mathbb{D},{ }^{15}$ Anding $X u \mathbb{D}^{1},{ }^{1}$ and Lingzhang Meng ${ }^{8}{ }^{8}$
}

${ }^{1}$ Jinan University, Guangzhou City, Guangdong Province, China

${ }^{2}$ Department of Neurology, Affiliated Hospital of Youjiang Medical University for Nationalities, Baise City, Guangxi Province, China ${ }^{3}$ Graduate School of Youjiang Medical University for Nationalities, Baise City, Guangxi Province, China

${ }^{4}$ Department of Neurology, The First People's Hospital of Yulin, Yulin City, Guangxi Province, China

${ }^{5}$ Department of Inverventional Medicine, Affiliated Hospital of Youjiang Medical University for Nationalities, Baise City, Guangxi Province, China

${ }^{6}$ Life Science and Clinical Research Center, Affiliated Hospital of Youjiang Medical University for Nationalities, Baise City, Guangxi Province, China

${ }^{7}$ Department of Rehabilitation Medicine, Affiliated Hospital of Youjiang Medical University for Nationalities, Baise City, Guangxi Province, China

${ }^{8}$ Center for Systemic Inflammation Research (CSIR), School of Preclinical Medicine, Youjiang Medical University for Nationalities, Baise City, Guangxi Province, China

${ }^{9}$ Urinary Surgery Department, Affiliated Hospital of Youjiang Medical University for Nationalities, Baise City, Guangxi Province, China

${ }^{10}$ College of Pharmacy, Youjiang Medical University for Nationalities, Baise City, Guangxi Province, China

${ }^{11}$ Trauma Center, Affiliated Hospital of Youjiang Medical University for Nationalities, Baise City, Guangxi Province, China

${ }^{12}$ Department of Biochemistry and Molecular Biology, Youjiang Medical University for Nationalities, Baise City, Guangxi Province, China

${ }^{13}$ Department of Gynaecology and Obstetrics, Affiliated Hospital of Youjiang Medical University for Nationalities, Baise City, Guangxi Province, China

${ }^{14}$ Shangsi People's Hospital, Fangchenggang City, Guangxi Province, China

${ }^{15}$ Department of Hepatological Surgery, Affiliated Hospital of Youjiang Medical University for Nationalities, Baise City, Guangxi Province, China

Correspondence should be addressed to Anding Xu; tlil@jnu.edu.cn and Lingzhang Meng; lingzhang.meng@ymcn.edu.cn

Received 13 September 2020; Revised 15 October 2020; Accepted 19 October 2020; Published 26 November 2020

Academic Editor: Jian Song

Copyright (C) 2020 Lanqing Meng et al. This is an open access article distributed under the Creative Commons Attribution License, which permits unrestricted use, distribution, and reproduction in any medium, provided the original work is properly cited.

Stroke is a cerebrovascular disease that results in decreased blood flow. Although Panax notoginseng (PN), a Chinese herbal medicine, has been proven to promote stroke recovery, its molecular mechanism remains unclear. In this study, middle cerebral artery occlusion (MCAO) was induced in rats with thrombi generated by thread and subsequently treated with PN. After that, staining with 2,3,5-triphenyltetrazolium chloride was employed to evaluate the infarcted area, and electron microscopy was used to assess ultrastructural changes of the neurovascular unit. RNA-Seq was performed to determine the differential expressed 
genes (DEGs) which were then verified by qPCR. In total, 817 DEGs were identified to be related to the therapeutic effect of PN on stroke recovery. Further analysis by Gene Oncology analysis and Kyoto Encyclopedia of Genes and Genomes revealed that most of these genes were involved in the biological function of nerves and blood vessels through the regulation of neuroactive live receptor interactions of PI3K-Akt, Rap1, cAMP, and cGMP-PKG signaling, which included in the 18 pathways identified in our research, of which, 9 were reported firstly that related to PN's neuroprotective effect. This research sheds light on the potential molecular mechanisms underlying the effects of PN on stroke recovery.

\section{Introduction}

Stroke is a major cause of death in the world, and it can also lead to long-term disability [1]. Ischemic stroke is due to cerebral artery occlusion, which interrupts the blood supply of the brain, resulting in hypoxia and a lack of nutrients, proceeded by a series of complex pathological changes. A common feature of ischemic stroke is cerebral ischemia and reperfusion injury (CIRI) which results in serious damage (Eltzschig and Eckle, 2011), including pathological processes such as excitatory glutamate toxicity, energy failure, free radical formation, oxidative stress, inflammatory response, $\mathrm{Ca}^{2+}$ overload, and apoptosis [2]. The concept of neurovascular unit (NVU) was first recognized in 2001, and alterations in the composition of the NVU have been shown to increase vulnerability to the damaging effects of ischemic stroke [3]. While the NVU has become an integral component in the study of biomarkers of ischemic stroke [4], effective neuroprotective drug targets in CIRI are yet to be determined.

Panax notoginseng is a precious Chinese herbal medicine, which is grown mainly in Wenshan Prefecture, Yunnan Province, China. It has been reported that at least twenty saponins were contained in this material, including notoginsenoside R1, ginsenoside $\mathrm{Rb} 1$, and ginsenoside $\operatorname{Rg} 1[5,6]$. It is anti-inflammatory and antioxidative, and it is able to regulate the balance of neurotransmitters and promoting the regeneration of nerves and blood vessels [7-9]. Our previous studies have shown that Panax notoginseng saponins (PNS), an extract of PN, have both antiinflammatory and neuroprotective effects [10]. Previously, it has been reported that PN exerted neuroprotective effect in stroke through antioxidative and anti-inflammatory properties [11]; in combination with Angelica sinensis, it could also inhibit NF- $\kappa B$ signaling and DNA binding activity, downregulate NO, NLRP3 inflammasome formation, and influence microglial pyroptosis $[12,13]$. PN may also influence the expression of Nogo- $\mathrm{A}, \mathrm{NgR}$, and p75, regulate NgR1/RhoA/Rock2 pathway, thus contribute to the recovery of nerve function in stroke $[14,15]$. However, further studies need to be conducted to explore underlying mechanism(s) of PN's protective effects on NVU, especially in stroke.

In this study, we first evaluated the effect of $\mathrm{PN}$ on the ultrastructure of the NVU, and then used the RNAsequencing methods to study the gene expression changes caused by $\mathrm{PN}$ on the brain ischemia-reperfusion injury rats. The differentially expressed genes (DEGs) and their signal pathways were also examined in an attempt to gain a better understanding of all the biochemical mechanisms involved in this process.

\section{Materials and Methods}

2.1. Animal and Experimental Design. Sprague-Dawley (SD) female rats $(250 \pm 30 \mathrm{~g})$ were purchased from Changsha Tianqin Biotechnology Co., Ltd, (Hunan, China). Before the experiments, all rats were maintained under standard laboratory conditions (a 12:12 hours of day and night cycle, a relative humidity of $60 \pm 5 \%$ at room temperature of $22 \pm 2^{\circ} \mathrm{C}$ and free access to food and water). This research was approved by the Animal Experimental Ethics Committee of Youjiang Medical University for Nationalities.

The middle cerebral artery occlusion (MCAO) model has been described in detail [16]. Briefly, we exposed the right internal and external carotid arteries; then, we cut external carotid artery about $3 \mathrm{~mm}$ above the common carotid artery bifurcation. After a silk suture was tied around the external carotid stump, a nylon filament (diameter: $0.265 \mathrm{~mm}$, rounded tip, dipped in heparin) was then inserted into the external carotid artery and gently advanced into the internal carotid artery, $17-19 \mathrm{~mm}$ from the carotid bifurcation until a detection of slight resistance. A nylon thread was then tied into the vascular lumen. After sterilization, suture incision and following $2 \mathrm{~h}$ of ischemia, the thrombus was pulled out for about $1 \mathrm{~cm}$ to complete reperfusion injury. Rats in the sham group underwent the same surgical procedures except for the nylon thread procedure. The ambient temperature was kept constant by maintaining the rectal temperature in the rats at $37 \pm 1^{\circ} \mathrm{C}$.

After being allowed to adapt to the environment for 7 days, at least 10 animals were randomly distributed in each group. The intervention group was administrated with PN (Sigma-Aldrich, \#1291719) intragastrically at a dose of $100 \mathrm{mg} \cdot \mathrm{kg}$ - 1 every 12 hours. The dose in this study was chosen based on previous study [13] and our preliminary experiments. Basically, 3 groups were designed in our study: the Sham group, the MCAO group which treated with saline for 7 days, and the MCAO group which treated with PN for 7 days. Both saline and PN administration started 2 hours after surgery. All rats were fasted but allowed free access to water.

\subsection{Evaluation of Neurological Defects. Neurobehavioral} impairment of rats was assessed in a double-blind manner by two independent observers according to Longa's [16]. Detailed information of this evaluation method was summarized in the supplementary table (available here).

The rats with 1-3 points were considered as successful modeling for I/R injury and were scored again for validation 7 days after surgery.

After 7 days, 6 rats were selected from each group and anesthetized. The rats in this study were anesthetized by IP injection of Ketamine $(80 \mathrm{mg} / \mathrm{kg})$ and Xylazine $(8 \mathrm{mg} / \mathrm{kg})$; 
supplemental heat was provided to avoid hypothermia during anesthesia, according to IACUC guidelines [17, 18]. Brain tissue was separated rapidly, and the area of cerebral infarction was measured using 2,3,5-triphenyltetrazolium chloride (TTC) staining in order to show the impaired area of cerebral ischemia.

2.3. Ultrastructural Changes of NVU [19, 20]. 7 days after the procedure, the cortex around the ischemic focus $(1 \mathrm{~mm} \times 1 \mathrm{~mm} \times 1 \mathrm{~mm})$ was immersed in $2.5 \%$ glutaraldehyde for 2-4hours and washed 3 times with $0.1 \mathrm{M}$ phosphate buffer ( $\mathrm{pH} 7.4$ ) for 15 minutes. The samples were fixed in osmic acid for 2 hours at room temperature, washed 3 times with $0.1 \mathrm{M}$ phosphate buffer ( $\mathrm{pH} 7.4)$ for 15 minutes, and fixed and stored at $4^{\circ} \mathrm{C}$. Subsequently, samples were dehydrated in an alcohol gradient $(50 \%-70 \%-$ 80\%-90\%-95\%-100\%-100\% ethanol-100\% acetone-100\% acetone) and embedded in EPON 812 epoxy resin. Ultrathin sections of $50 \mathrm{~nm}$ were stained with $3 \%$ saturated solution of uranyl acetate and 6\% lead citrate staining solution. The ultrastructure of neurons, astrocytes, and endothelial cells was observed using a JEOL-1011 transmission electron microscope (JEOL, Japan) at $80 \mathrm{kV}$.

2.4. RNA Extraction, Library Construction, and Sequencing. According to the manufacturer's protocol, total RNA was extracted with Trizol kit (Invitrogen, USA). RNA quality was evaluated on the Agilent 2100 Bioanalyzer (Agilent Technologies, USA) and verified by RNase-free agarose gel electrophoresis. After extraction, oligo (dT) beads were used to enrich eukaryotic mRNA; then, Ribo-ZeroTM magnetic kit (Epicentre, USA) was used to remove rRNA.

The mRNA acquired above was treated with fragmentation buffer, then reverse transcribed into cDNA. Secondstrand cDNA was synthesized with DNA polymerase I, RNase $\mathrm{H}$, and $\mathrm{dNTP}$, and then purified with a QiaQuick PCR extraction kit (Qiagen, Netherlands); end-repaired, poly(A) tails were added and ligated to Illumina sequencing adapters. The ligation products were separated and selected based on size with agarose gel electrophoresis, and PCR amplification and sequencing were performed with Illumina HiSeq2500.

2.5. Assembling and Processing Sequencing Raw Data. Clean readings were obtained by removing reads which contain adapters, those with more than $10 \%$ of unknown nucleotides (N) and low-quality reads containing more than $50 \%$ of low quality $(Q$ value $\leq 20)$ bases. Using StringTie v1.3.1 $[21,22]$, transcripts were assembled from sequenced raw data processed by the HISAT2. 2.4 [23] method. For each transcription region, a fragment per kilobase of transcript per million mapped reads (FPKM) [24] value was calculated to quantify its expression abundance and variation, using the StringTie software.

2.6. Differentially Expressed Genes. RNAs differential expression analysis was performed by using DESeq2 [25] software between two different groups (and by edge $\mathrm{R}$ [26] between two samples). The genes with a false discovery rate (FDR) below 0.05 and an absolute fold-change $\geq 2$ were considered


Sham

Saline

PN

MCAO

FIgURe 1: Brain tissue samples of rats from each group. (a) Sham group, (a1) cerebral vessels are abundant and clearly visible, (a2) brain tissue is full and symmetrical. (b) MCAO group treated with saline, (b1) blood vessels around the infarction show exudation, blocked blood vessels are seen to be collapsed and atrophied, and (b2) brain tissue is swollen and white. (c) MCAO group treated with $\mathrm{PN},(\mathrm{c} 1)$ blood vessels around the infarction are mostly new, with less exudation than MCAO group and (c2) brain tissue is not swollen obviously. The yellow lines indicate the infarct area. Similar experiments were performed at least 5 times.

to be DEGs. qPCR was performed in order to verify the expression of these screened DEGs between the different groups.

2.7. Validation of the RNA-Sequencing Results by qPCR. To verify the reliability of sequencing results, qPCR was used to verify gene expression in the same batches of MCAO and PN samples $(n=6)$, and $\beta$-actin was used as an internal reference. The $20 \mu \mathrm{L}$ reaction system contained $5 \mu \mathrm{L}$ of cDNA, $0.5 \mu \mathrm{L}$ of each primer, $0.5 \mu \mathrm{L} 2 \times$ SYBR Green qPCR

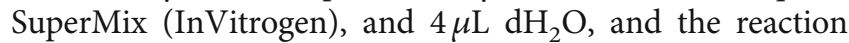
conditions were as follows: $50^{\circ} \mathrm{C}$ for $2 \mathrm{~min}, 95^{\circ} \mathrm{C}$ for $2 \mathrm{~min}$, $95^{\circ} \mathrm{C}$ for $15 \mathrm{~s}$, and $60^{\circ} \mathrm{C}$ for $32 \mathrm{~s}$ plate read for 40 cycles followed by melting curve analysis $\left(60^{\circ} \mathrm{C}\right.$ to $\left.95^{\circ} \mathrm{C}\right)$. The $2^{-\triangle \Delta \mathrm{Ct}}$ method was used to determine the relative amount of mRNA, and 3 measurements were made for each sample. Primers used for Cttn amplification are $5^{\prime}$-ATGTGGAAA GCTTCAGCAGGCC- $3^{\prime}$ (forward) and $5^{\prime}$-TCACGGGCA CT CCGGGACCCAA-3' (reversed); primers used for Cxcl1 amplification are $5^{\prime}$-AAATGGTGAAGGTCGGTGTGAA$3^{\prime}$ (forward) and $5^{\prime}$-CAACAATCTC CACTTTGCCACTG$3^{\prime}$ (reversed); primers used for Snap25 amplification are $5^{\prime}$ -AGGACTTTGGTTATGTTGGAT-3' (forward) and $5^{\prime}$ -GATTTAAGCTTGTT ACAGG-3' (reversed); primers used for Nox1 amplification are $5^{\prime}$-CTTTAGCATCCATA TCCGCATT- $3^{\prime}$ (forward) and $5^{\prime}$-GACTGGTGGCATTG 




(a)

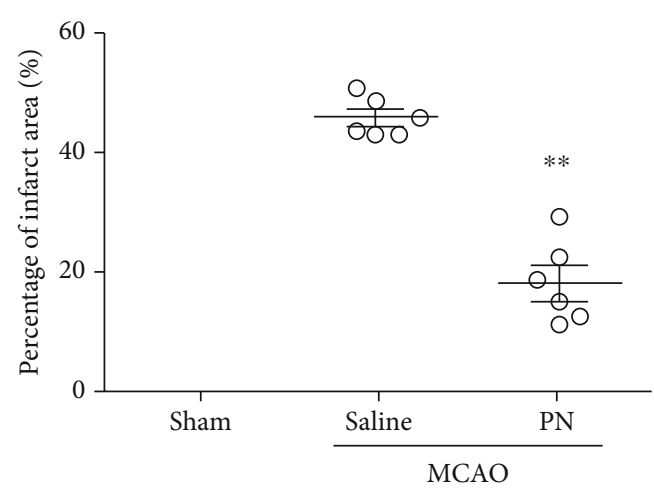

(c)

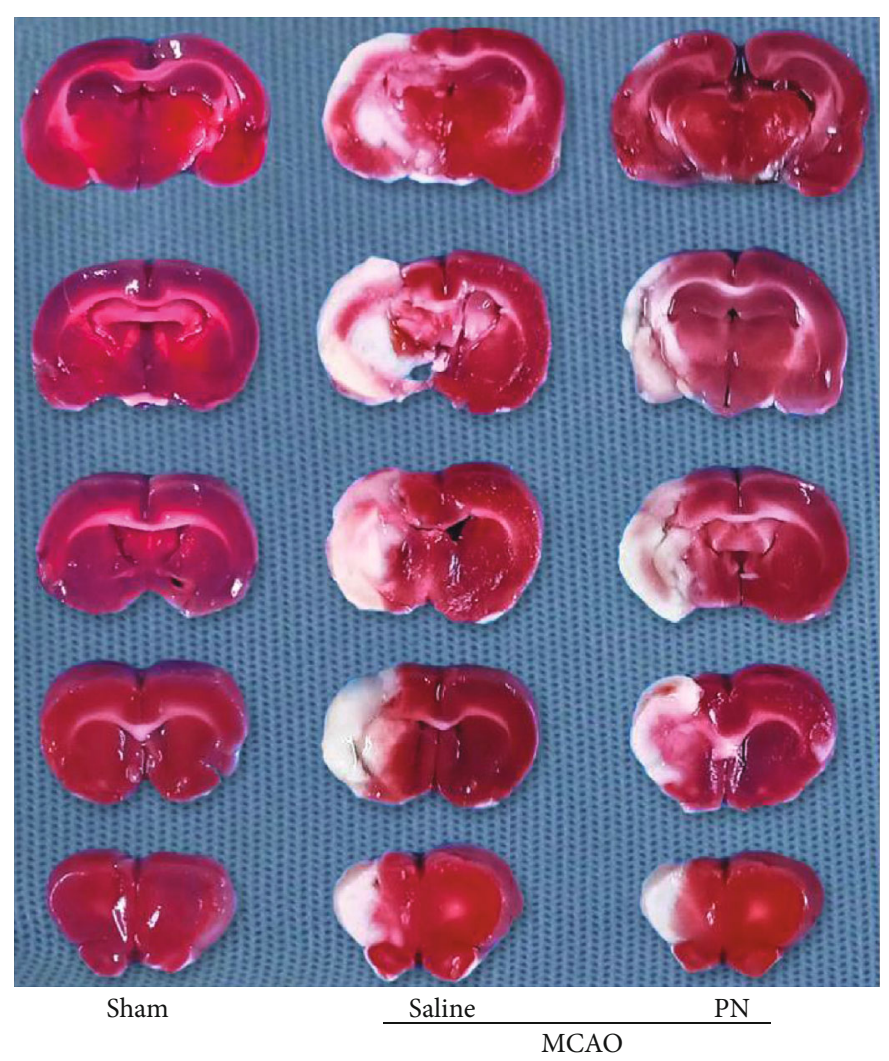

(b)

Figure 2: The effects of PN on MACO in rat infarction volume. (a) Compared with the MCAO group treated with saline, scores of neural function were decreased in the PN group ( $n=10$ in each group). (b) TTC staining, normal tissues are red and infarcted tissues are white. PN can effectively reduce the range of cerebral infarction in rats. (c) Compared with MCAO group, the infarct area and percentage in the PN group decreased significantly ( $n=6$ in each group). In (a) and (c), data shown indicated as mean \pm SEM values. Similar experiments were performed at least 3 times. ${ }^{*} P<0.05 ;{ }^{* *} P<0.01 ;{ }^{* * *} P<0.001$.
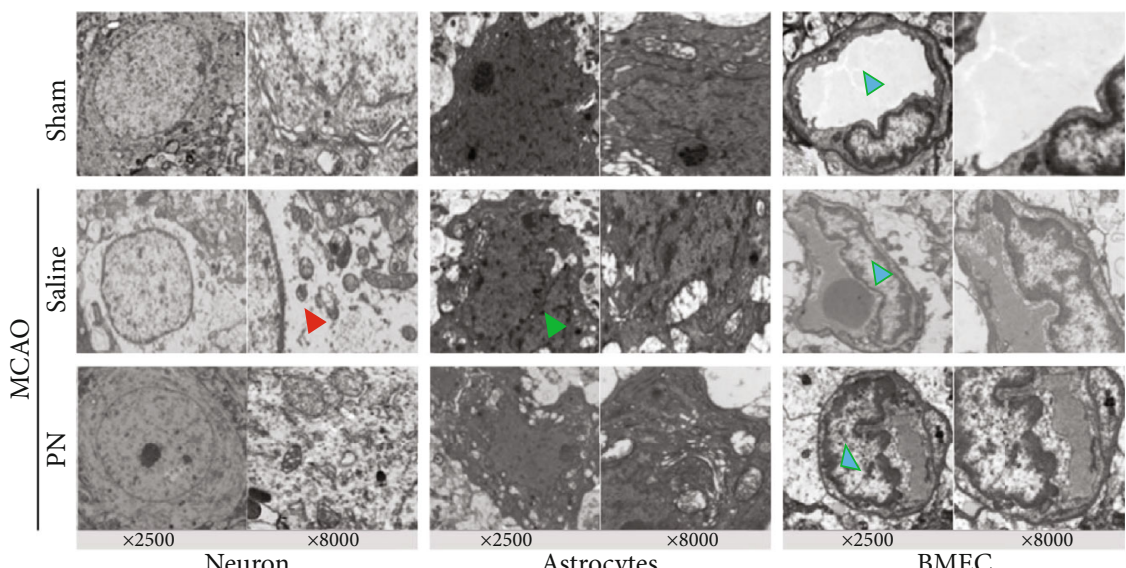

FIGURE 3: The effect of PN on ultrastructural changes of NVU. Similar experiments were performed at least 4 times. BMEC represents brain microvascular endothelial cell. The red triangle indicates a severe swollen/inflammation around neuron, the green triangle indicates an irregular nucleus. The blue triangle indicates the lumen side of brain microvascular.

TC ACAATA-3' (reversed); primers used for Bcl2 amplification are $5^{\prime}$-ACGAGTGG GATACTGGAGATG-3' (forward) and $5^{\prime}$-TAGCGACGAGAGAAGTCATCC-3' (reversed); primers used for Kdr amplification are $5^{\prime}$-TCACGGTTGGG CTACTGC-3' (forward) and $5^{\prime}$-AGACCTTCTGCCATCA CG-3' (reversed); primers used for Foxo3 amplification are 


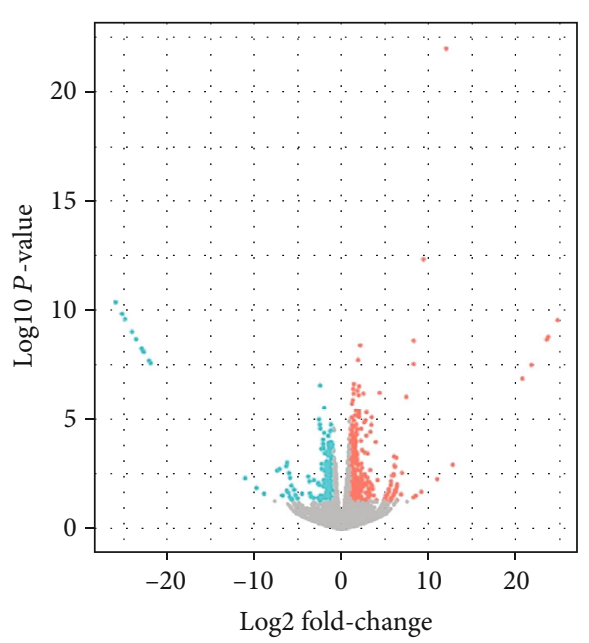

Threshold

- Down (427)

- Maintain (20783)

- Up (390)

(a)

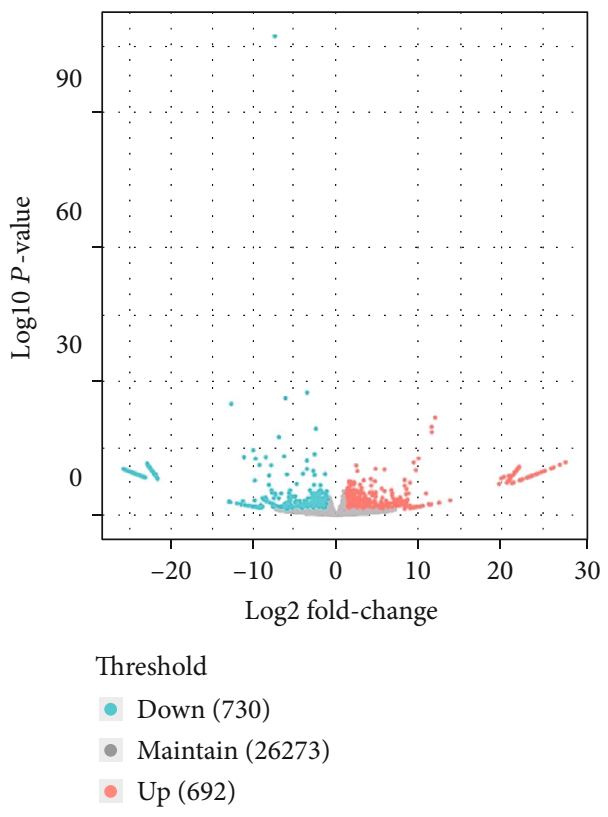

(c)

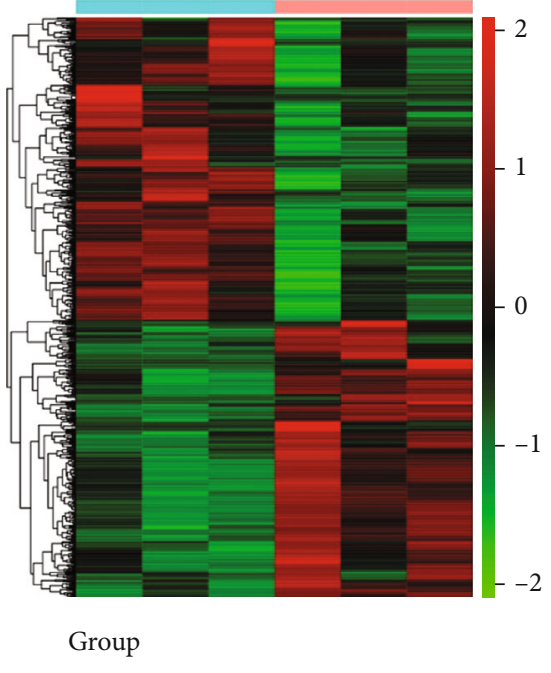

MCAO (Saline)

$\mathrm{MCAO}(\mathrm{PN})$ (b)

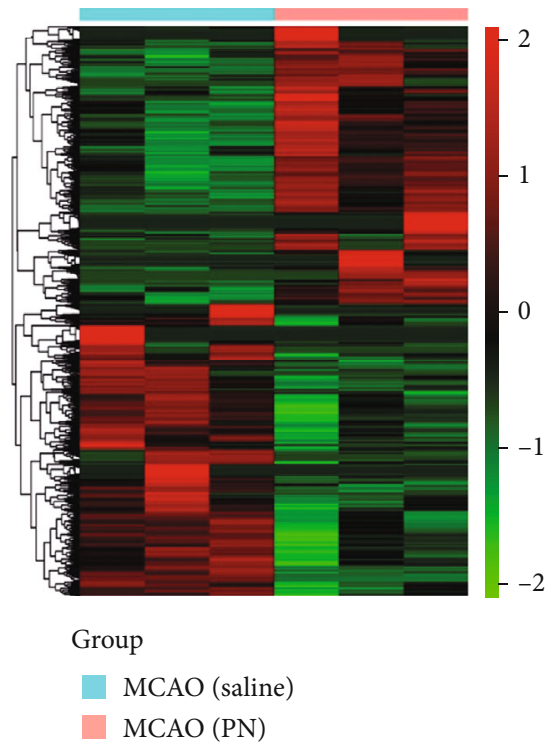

(d)

FIGURE 4: Volcano plots and hierarchical clustering heat map of DEGs and DETs. (a) Volcano map for DEGs where red represents upregulated genes and green represents downregulated genes. (b) Heat map for hierarchical clustering of DEGs. (c) Volcano map for DETs, red represents upregulated transcripts, and green represents downregulated transcripts. (d) Heat map for hierarchical clustering of DETs). $n=3$ in each group.

$5^{\prime}$-TGGATGACCTGCTAGATAACAT-3' (forward) and 5' -AACACGGTACTGTTAAAGGAGC-3' (reversed); primers used for Notch3 amplification are $5^{\prime}$-GCTGGCGTCTCTTC AACAACA- ${ }^{\prime}$ (forward) and $5^{\prime}$-TGGTCGGCGCAGTACT TCTTAT- $3^{\prime}$ (reversed); primers used for $\beta$-actin (reference gene used in this study) amplification are $5^{\prime}$-AGGGAAATCG
TGCGTGACAT-3' (forward) and $5^{\prime}$-GAACCGCTCATTGC CGATAG-3' (reversed).

2.8. Enrichment Analysis of DEGs. In order to understand the biological functions and pathways of DEGs in rat cerebral ischemia after PN intervention, we used an annotation and 
TABLE 1: The partially DEGs from RNA-Seq $[\mathrm{MCAO}($ Saline)/MCAO(PN)].

\begin{tabular}{lccccc}
\hline Ensembl gene ID & Gene symbol & Log2-fold change & $P$ value & $P$ adj & Style \\
\hline ENSRNOG00000050994 & Cttn & 12.67967226 & 0.001172369 & 0.104286972 & Up \\
ENSRNOG00000002802 & Cxcl1 & 2.948761024 & 0.007257485 & 0.253659663 & Up \\
ENSRNOG00000006037 & Snap25 & 1.675988534 & 0.0003127 & 0.046747614 \\
ENSRNOG00000048706 & Nox1 & 1.96651 & 0.011922266 & 0.31373928 & Up \\
ENSRNOG00000002791 & Bcl2 & -1.777692036 & 0.003557504 & 0.176645472 & Down \\
ENSRNOG00000046829 & Kdr & -1.524693848 & 0.000278591 & 0.044899468 & Down \\
ENSRNOG00000000299 & Foxo3 & -1.159297737 & 0.035026006 & 0.476124441 & Down \\
ENSRNOG00000004346 & Notch3 & -1.450412508 & 0.014108102 & 0.337843688 & Down \\
\hline
\end{tabular}

TABLE 2: The partially DETs from RNA-Seq [MCAO(Saline)/MCAO(PN)].

\begin{tabular}{lcccccc}
\hline Transcript ID & Gene ID & Gene symbol & Log2-fold change & $P$ value & $P$ adj & Style \\
\hline ENSRNOT00000000508 & ENSRNOG00000000439 & Ager & 3.050138008 & 0.022180829 & 0.401239754 & Up \\
ENSRNOT00000000553 & ENSRNOG00000000473 & Pfdn6 & 2.208199784 & 0.002663896 & 0.121543007 & Up \\
ENSRNOT00000000657 & ENSRNOG00000000546 & Nt5dc1 & 1.080452666 & 0.036038318 & 0.50948505 & Up \\
ENSRNOT00000000817 & ENSRNOG00000000657 & Nek7 & 3.035450677 & 0.001253867 & 0.070869068 & Up \\
ENSRNOT00000002487 & ENSRNOG00000001816 & Rfc4 & 1.358324579 & 0.00530658 & 0.182793209 & Up \\
ENSRNOT00000000205 & ENSRNOG00000024631 & Chadl & -1.127386831 & 0.006102632 & 0.202410056 & Down \\
ENSRNOT0000000824 & ENSRNOG00000000661 & Hps4 & -2.446591496 & 0.004995283 & 0.177592248 & Down \\
ENSRNOT00000001185 & ENSRNOG00000000886 & Caln1 & -1.460830778 & 0.015749777 & 0.338238335 & Down \\
ENSRNOT00000001248 & ENSRNOG00000000940 & Flt1 & -1.41782469 & 0.001002321 & 0.059826058 & Down \\
ENSRNOT00000001479 & ENSRNOG00000001117 & Fbxl18 & -1.477639058 & 0.020547561 & 0.386592868 & Down \\
\hline & & & &
\end{tabular}

visualization integrated OmicShare cloud platform to analyze and visualize the biological functions and pathways of differential genes.

2.9. Statistical Analysis. All data are expressed as mean \pm SEM, and statistical analysis was performed using the SPSS 22.0 statistical software (IBM, Chicago, Illinois). The experimental data were analyzed by one-way analysis of variance. Student's paired $t$-test was used to compare qPCR results, and $P$ values $<0.05$ were considered statistically significant. GO Analysis was based on the GO database. Fisher's exact test and multiple comparison test were used to calculate the significance level ( $P$ value) and false positive rate (FDR) of each function. $P$ value $<0.05$ was the criterion for significance screening. Pathway analysis was based on the KEGG database, and Fisher's exact test and chi-square test were used for the DEGs. A pathway in which the target gene participated in was analyzed for significance and selected according to $P$ value $<0.05$

\section{Results}

3.1. The Effects of PN on MCAO in Rats Infarction Volume. In order to evaluate the protective effect of PN on cerebral ischemia, on the seventh day of the experiment, the neurological functions were assessed blindly using the Longa Neurological Severity Score; the brain was removed after anesthesia (Figure 1) and subjected to TTC staining in order to evaluate the scope of infarction (Figure 2). Comparing with the MCAO group treated with saline, PN-treated animals showed decreased disease score (Figure 2(a)) and significantly less infarct area (Figures 2(b) and 2(c)).

3.2. Ultrastructural Regulation of the NVU after PN Treatment. Ultrastructural changes in the NVU were observed after 7 days of cerebral ischemia. Figure 3 shows in the Sham group the neurons, and astrocytes are clearly visible and have large and rounded nuclei. The microvascular endothelial cells are clear structures without edema around them, and the lumen is normal. At high magnification, the binuclear membrane appears clear and complete with a clear field of view of the integrity of the cell structure. Organelles, such as lysosomes, are distributed throughout the cytoplasm. The surface of vascular endothelial cells is smooth and flat; and the endothelium, basement membrane, and foot processes are in close contact.

In the MCAO group, the neurons and astrocytes are irregular and show an accumulation of chromatin. Cytoplasm lysis and vacuole formation were observed. There appears to be estrogen receptor expansion, mitochondrial bending, disorder, shrinkage, and vacuolation in the cytoplasm. Mitochondrial and other organ-related injuries were more severe than those in the treatment group. Edema around blood vessels is obvious, showing vacuoles or blank areas (Figure 3).

In the PN group, the nuclei of neurons appear more normal with the nuclear membrane being intact, and the nucleoli are visible. The astrocyte structure is more complete than in the MCAO group with the nuclear morphology being more complete and clear, the microvascular endothelial cells are 




(a)



(b)

FiguRE 5: Verification of DEGs obtained from RNA-Seq by qPCR. Significance level according to nonparametric test (Cruskal-Wallis test, $n=6$ in each group). Data shown indicated as mean \pm SEM values. Similar results were obtained from at least 3 independent experiments. ${ }^{*} P<0.05 ;{ }^{* *} P<0.01 ;{ }^{* * *} P<0.001$.

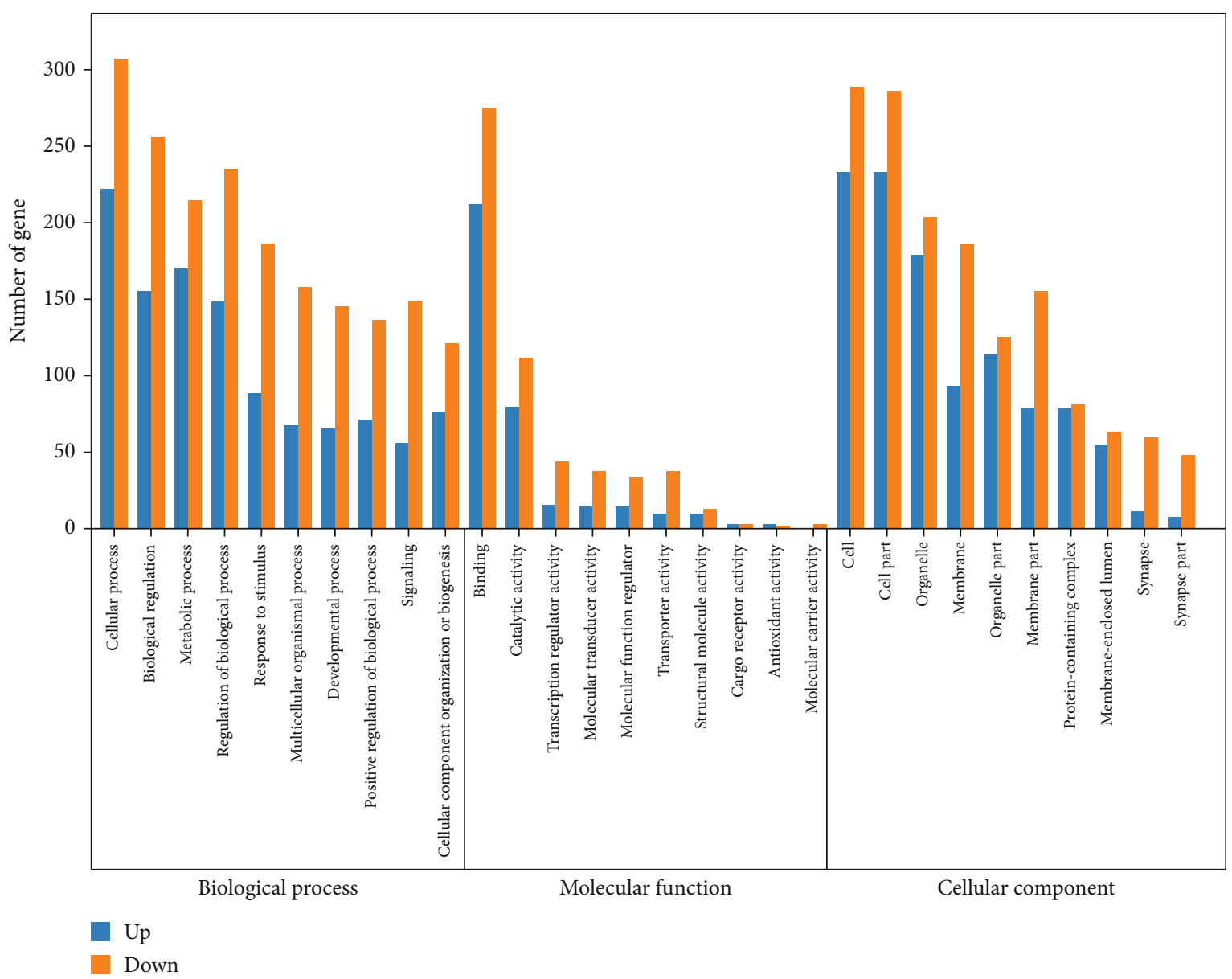

FIGURE 6: Gene ontology enrichment analysis of DEGs. The ordinate is the number of genes, the abscissa is the GO name. Blue refers to the upregulated genes; orange refers to the downregulated genes.

not deformed significantly, and the lumen is basically unobstructed. The peripheral edema is reduced compared with the MCAO group (Figure 3).
3.3. Gene Expression and Transcript Data Analysis. To investigate the molecular mechanism of the protective effect of PN on MCAO in rats, brain tissue gene expression profiles of the 
TABLE 3: GO classification of DEGs in MCAO(Saline)/MCAO(PN) (Top30).

\begin{tabular}{|c|c|c|c|c|}
\hline Term & Description & Type & Count & $P$ value \\
\hline GO:0031323 & Regulation of cellular metabolic process & $\mathrm{BP}$ & 218 & $2.85 \mathrm{E}-06$ \\
\hline GO:0001568 & Blood vessel development & $\mathrm{BP}$ & 40 & $2.99 \mathrm{E}-06$ \\
\hline GO:0001944 & Vasculature development & $\mathrm{BP}$ & 41 & $3.22 \mathrm{E}-06$ \\
\hline GO:0006836 & Neurotransmitter transport & $\mathrm{BP}$ & 23 & $3.64 \mathrm{E}-06$ \\
\hline GO:0080090 & Regulation of primary metabolic process & $\mathrm{BP}$ & 213 & $3.85 \mathrm{E}-06$ \\
\hline GO:0019222 & Regulation of metabolic process & $\mathrm{BP}$ & 230 & $3.92 \mathrm{E}-06$ \\
\hline GO:0065008 & Regulation of biological quality & $\mathrm{BP}$ & 153 & $4.21 \mathrm{E}-06$ \\
\hline GO:0072358 & Cardiovascular system development & $\mathrm{BP}$ & 41 & 4.92E-06 \\
\hline GO:0050905 & Neuromuscular process & $\mathrm{BP}$ & 14 & $5.87 \mathrm{E}-06$ \\
\hline GO:0007626 & Locomotory behavior & $\mathrm{BP}$ & 20 & $6.12 \mathrm{E}-06$ \\
\hline GO:0003676 & Nucleic acid binding & MF & 163 & $1.31 \mathrm{E}-06$ \\
\hline GO:0097159 & Organic cyclic compound binding & MF & 233 & $1.29 \mathrm{E}-05$ \\
\hline GO:1901363 & Heterocyclic compound binding & MF & 230 & $1.40 \mathrm{E}-05$ \\
\hline GO:0046872 & Metal ion binding & MF & 138 & $1.89 \mathrm{E}-05$ \\
\hline GO:0043169 & Cation binding & MF & 141 & $2.04 \mathrm{E}-05$ \\
\hline GO:0005488 & Binding & MF & 488 & $5.61 \mathrm{E}-05$ \\
\hline GO:0016263 & Ion binding & MF & 200 & 0.002322647 \\
\hline GO:0043565 & Sequence-specific DNA binding & MF & 53 & 0.001285424 \\
\hline GO:0003677 & DNA binding & MF & 85 & 0.001632778 \\
\hline GO:0003690 & Double-stranded DNA binding & MF & 44 & 0.001941181 \\
\hline GO:0045202 & Synapse & $\mathrm{CC}$ & 70 & $3.86 \mathrm{E}-08$ \\
\hline GO:0044456 & Synapse part & $\mathrm{CC}$ & 55 & $2.90 \mathrm{E}-06$ \\
\hline GO:0098794 & Postsynapse & $\mathrm{CC}$ & 40 & $4.36 \mathrm{E}-06$ \\
\hline GO:0098984 & Neuron to neuron synapse & $\mathrm{CC}$ & 23 & 0.000185124 \\
\hline GO:0005622 & Intracellular & $\mathrm{CC}$ & 454 & 0.000264158 \\
\hline GO:0045211 & Postsynaptic membrane & $\mathrm{CC}$ & 20 & 0.000272495 \\
\hline GO:0097060 & Synaptic membrane & $\mathrm{CC}$ & 25 & 0.000310002 \\
\hline GO:0098978 & Blutamatergic synapse & $\mathrm{CC}$ & 26 & 0.000423116 \\
\hline GO:0032279 & Asymmetric synapse & $\mathrm{CC}$ & 21 & 0.000463989 \\
\hline GO:0034707 & Chloride channel complex & CC & 6 & 0.000580336 \\
\hline
\end{tabular}

*BP: biological process; MF: molecular function; CC: cellular component; Count: gene number listed in GO term.

Sham, MCAO, and PN groups were measured by using RNA-Seq. A total of 817 DEGs were observed in MCAO group compared to PN group, of which, 390 genes were upregulated and 427 were downregulated (Figures 4(a) and 4(b), Table 1). In addition, there were 1422 differentially expressed transcripts (DETs) which included 692 upregulated and 730 downregulated transcripts (Figures 4(c) and 4(d), Table 2).

3.4. RNA-Seq Validation by qPCR. To verify the expression of the DEGs obtained from the RNA-Seq results between MCAO and PN groups, we randomly selected 8 genes for validation, including 4 upregulated genes (Cttn, Cxcl1, Snap25, and Nox1) and 4 downregulated genes ( $\mathrm{Bcl} 2, \mathrm{Kdr}$, Foxo3 and Notch3). The relative expression was determined by the $2^{-\triangle \Delta \mathrm{Ct}}$ method, and the results were consistent with the expression trends seen during RNA-Seq analysis (Figure 5). The qPCR results were consistent with the sequencing experiments.
3.5. GO and Pathway Analyses. In order to determine the functions and pathways of DEGs and DETs related to PN treatment, we performed GO and pathway analysis. Using $P<0.01$ identified 332 GO terms, 202 of which were related to nerves and 55 were related to blood vessels, of which the following GO terms: cellular process (GO:0009987), biological regulation (GO:0065007), metabolic process (GO:0008152), regulation of biological process (GO:0050789), response to stimulus (GO:0050896), binding (GO:0005488), catalytic activity (GO:0003824), transcription regulator activity (GO:0140110), molecular transducer activity (GO:0060089), molecular function regulator (GO:0098772), cell (GO:0005623), cell part (GO:0044464), organelle (GO:0043226), membrane (GO:0016020), and organelle part (GO:0044422) were significantly different between saline and PN-treated groups. This indicates that the above biological processes are potentially related to the therapeutic function of PN. 


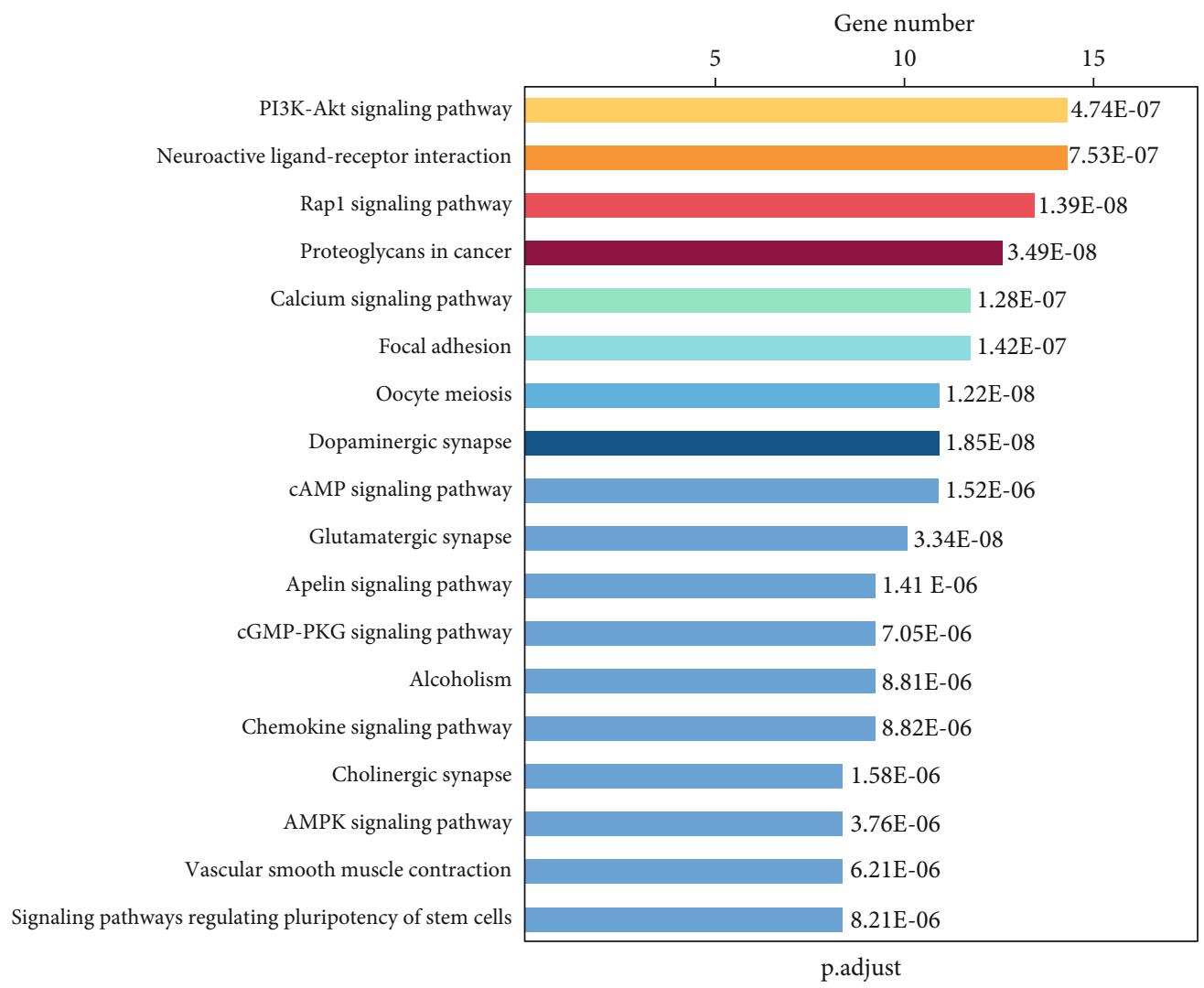

FIGURE 7: The list of top 18 enriched pathway analysis of DEGs.

According to the enrichment degree of functional annotation, the top 10 biological processes, molecular functions, and cellular composition are shown in Figure 6 and Table 3. KEGG pathway analysis leads to a better understanding of the role of PN in the treatment of cerebral ischemia. 39 pathways were screened with $P$ values of less than 0.05 , of which 18 pathways were enriched with more than 10 genes. These include PI3K-Akt (ko04151), Rap1 (ko04015), neuroactive ligand-receptor interaction (ko04080), calcium (ko04020), focal adhesion (ko04510), oocyte meiosis (ko04114), cAMP (ko04024), glutamatergic synapse (ko04724), dopaminergic synapse (ko04728), apelin (ko04371), cGMP PKG (ko04022), chemokine (ko04062), cholinergic synapse (ko04725), vascular smooth muscle contraction (ko04270), AMPK (ko04152), and signaling pathways regulating pluripotency of stem cells (ko04550) (Figure 7), and the genes participated in the above pathways were listed in Table 4 . These pathways and genes may play an important role in the antiischemic effect of PN (Figure 8).

\section{Discussion}

It has been proven that $\mathrm{PN}$ possesses therapeutic properties against stroke, and it has been reported in previous studies about the molecular mechanism(s) explaining PN's clinical outcomes [11-13]. However, most of previous research focused on single a gene/pathway, still no systemic evaluation of PN's molecular mechanism(s) in neuroprotection effect.
In this case, based on a rat stroke model treated with PN, we employed the second generation sequencing technology, which is a high-throughput method, in combination of qPCR and transmission electron microscopy; this study identified 817 DEGs potentially related to PN's neuroprotection effect, and we firstly identified 18 pathways involved in its therapeutic effect.

The development of stroke involves energy metabolism disorder, inflammatory response, free radical formation, calcium overload and apoptosis, and destruction of blood-brain barrier [2]. These adverse factors cause pathological damage to neurons, glial cells, and microvessels in the NVU [27].

In this study, we investigated the protective effect of PN on ischemic stroke in rats. Compared with the MCAO model group, PN treatment can significantly decrease the infarct volume/size in rats with cerebral ischemia and reduce the volume of cerebral infarction as shown by TTC staining. Hence, $\mathrm{PN}$ has an anti-ischemic effect. Ultrastructural results show that PN can reduce the pathological changes of the NVU in ischemic stroke.

KEGG pathway analysis showed PN's therapeutic effect involves various pathways such as PI3K-Akt pathway, Neuroactive ligand-receptor interaction, Rap1 signaling pathway, proteoglycans in cancer, calcium signaling pathway, focal adhesion, oocyte meiosis, cAMP signaling pathway, Glutamatergic synapse, Dopaminergic synapse, Apelin signaling pathway, cGMP-PKG signaling pathway, Alcoholism, Chemokine signaling pathway, Cholinergic synapse, Vascular 
TABLE 4: A list of the top 18 KEGG pathway DEGs.

\begin{tabular}{|c|c|c|}
\hline Pathway ID & Pathway & Genes \\
\hline ko04151 & PI3K-Akt signaling pathway & $\begin{array}{l}\text { Lpar4, Col6a5, Ppp2r3c, Foxo3, Flt1, Bcl2, Gsk3b, Pdpk1,Erbb2, Epha2, Gnb4, Igf1r, } \\
\text { Fn1, Kdr, Chrm2, Itga1, Ppp2r2c. }\end{array}$ \\
\hline ko04080 & $\begin{array}{l}\text { Neuroactive ligand-receptor } \\
\text { interaction }\end{array}$ & $\begin{array}{l}\text { Rxfp2, Lpar4, Gcgr, Adora2a, Adora1, Apln, Gabrb2, Drd2, Grin2b, Grik3, Aplnr, } \\
\text { Adra1a, Oprd1, Ednrb, Cysltr2, Grin2a, Chrm2. }\end{array}$ \\
\hline ko04015 & Rap1 signaling pathway & $\begin{array}{l}\text { Lpar4, Map2k3, Flt1, Adora2a, Rapgef4, Adcy5, Drd2, Grin2b, Epha2, Igf1r, Plce1, } \\
\text { Rapgef1, Tln2, Grin2a, Kdr, Adcy1. }\end{array}$ \\
\hline ko05205 & Proteoglycans in cancer & $\begin{array}{l}\text { Rock1, Plaur, Cttn, Itpr2, Timp3, Pdpk1, Erbb2, Itpr1, Igf1r, Plce1, Fn1, Ank1, } \\
\text { Camk2a, Kdr, Fzd3. }\end{array}$ \\
\hline ko04020 & Calcium signaling pathway & $\begin{array}{l}\text { Ppp3r1, Adora2a, Itpr2, Itpkb, Erbb2, Itpr1, Adra1a,Ednrb, Plce1, Cysltr2, Camk2a, } \\
\text { Grin2a, Chrm2,A dcy1. }\end{array}$ \\
\hline ko04510 & Focal adhesion & $\begin{array}{c}\text { Col6a5, Rock1, Flt1, Bcl2, Gsk3b, Pdpk1, Erbb2, Igf1r, Fn1, Rapgef1, Shc3, Tln2, Kdr, } \\
\text { Itga1. }\end{array}$ \\
\hline ko04114 & Oocyte meiosis & $\begin{array}{l}\text { Rps6ka6, Pttg1, Spdya, Espl1, Fbxo5, Ppp3r1, Itpr2,Adcy5, Itpr1, Igf1r, Camk2a, } \\
\text { Cpeb3, Adcy1. }\end{array}$ \\
\hline ko04024 & cAMP signaling pathway & $\begin{array}{l}\text { Rock1, Cftr, Adora2a, Rapgef4, Adcy5, Adora1, Drd2, Grin2b, Plce1, Camk2a, Grin2a, } \\
\text { Chrm2, Adcy1. }\end{array}$ \\
\hline ko04724 & Glutamatergic synapse & $\begin{array}{c}\text { Ppp3r1, Arrb1,Itpr2,Adcy5, Itpr1, Grin2b, Grik3, Slc1a3, Shank1, Grin2a, Shank3, } \\
\text { Gnb4, Adcy1. }\end{array}$ \\
\hline ko04728 & Dopaminergic synapse & $\begin{array}{l}\text { Ppp2r3c, Itpr2, Adcy5, Gsk3b, Ddc, Itpr1, Drd2, Grin2b, Gnb4, Camk2a, Grin2a, } \\
\text { Ppp2r2c. }\end{array}$ \\
\hline ko04371 & Apelin signaling pathway & Itpr2, Adcy5, Apln, Notch3, Itpr1, Aplnr, Gnb4, Klf2, Hdac4, Acta2, Adcy1. \\
\hline ko04022 & cGMP-PKG signaling pathway & Rock1, Ppp3r1, Itpr2, Adcy5, Adora1, Itpr1, Adra1a, Oprd1, Ednrb, Gucyla2, Adcy1. \\
\hline ko05034 & Alcoholism & $\begin{array}{l}\text { Adora2a, Camkk2, Adcy5, Ddc, Drd2, Grin2b, Gnb4, Shc3, Hdac4, Grin2a, } \\
\text { LOC103690190. }\end{array}$ \\
\hline ko04062 & Chemokine signaling pathway & Cxcl1, Rock1, Foxo3, Adcy5, Gsk3b, Gnb4, Grk5, Cxcl12, Shc3, Arrb1, Adcy1. \\
\hline ko04725 & Cholinergic synapse & Itpr2, Adcy5, Bcl2, Kcnq3, Itpr1, Gnb4, Camk2a, Chrm2, Adcy1, Slc18a3. \\
\hline ko04270 & Vascular smooth muscle contraction & Rock1, Adora2a, Itpr2, Adcy5, Prkch, Itpr1, Adra1a, Gucy1a2, Acta2, Adcy1. \\
\hline ko04152 & AMPK signaling pathway & Ppp2r3c, Cftr, Foxo3, Camkk2, Pdpk1, Adipor2, Adra1a, Scd, Igf1r, Ppp2r2c. \\
\hline ko04550 & $\begin{array}{l}\text { Signaling pathways regulating } \\
\text { pluripotency of stem cells }\end{array}$ & Gsk3b, Tbx3, Skil, Lifr, Isl1, Igf1r, Bmpr1, Neurog1, Apc2, Fzd3. \\
\hline
\end{tabular}

smooth muscle contraction, and AMPK signaling pathway. Nine of which were firstly reported in this research to be related to $\mathrm{PN}$.

It has been reported that PN exerts different molecular mechanisms and pathways related to its neuroprotective effect. Panax notoginseng saponins are the representative bioactive agent of PN extracts, and it is widely used in the treatment of ischemic stroke, probably due to its inhibition of apoptosis via upregulation of SIRT1 and antioxidants [28]. PN and its extract are known to have a variety of protective neurovascular mechanisms. For example, PNS protects cerebral microvascular endothelial cells by activating the PI3K/Akt/Nrf2 antioxidant signal pathway [29]. Notoginsenoside R1 plays a neuroprotective role by activating the estrogen receptor-dependent Akt/Nrf2 pathway to inhibit NADPH oxidase activity and mitochondrial dysfunction [30]. Ginsenoside Rb1 can upregulate the expression of GDNF to inhibit neuron apoptosis [31] and promote motor function recovery and axon regeneration in mice after stroke through the cAMP/PKA/CREB signaling pathway [32]. Ginsenoside Rd has been shown to inhibit microglial proteasome activity and inflammatory response $[33,34]$ through mitochondrial protection, apoptosis inhibition, and energy recov- ery. Ginsenoside Rg1 can regulate the inhibition of NMDA receptor channels and L-type voltage-dependent calcium channels on $\mathrm{Ca}^{2+}$ influx and the decrease of intracellularfree $\mathrm{Ca}^{2+}$ caused by it, thus playing a neuroprotective role [2]. Studies have also shown that the cGMP-PKG pathway mediates the proliferation of neural stem cells after cerebral ischemia [35]. Ras-associated protein 1 (Rap1) is known to be involved in integrin and cadherin-mediated adhesion, which can mediate angiogenesis in endothelial cells [36].

In our research, we identified another 9 pathways potentially related to PN's neuroprotective effect, including Neuroactive ligand-receptor interaction, proteoglycans in cancer, focal adhesion, oocyte meiosis, Apelin signaling pathway, Alcoholism, Chemokine signaling pathway, Cholinergic synapse, and Vascular smooth muscle contraction.

Genetically modified animals should be used to confirm the biological/pathological role of these newly identified pathways related to PN's therapeutic effect against stroke.

\section{Conclusions}

The NVU is the structural and functional unit of the nervous system. Our study shows that PN has a protective effect on 


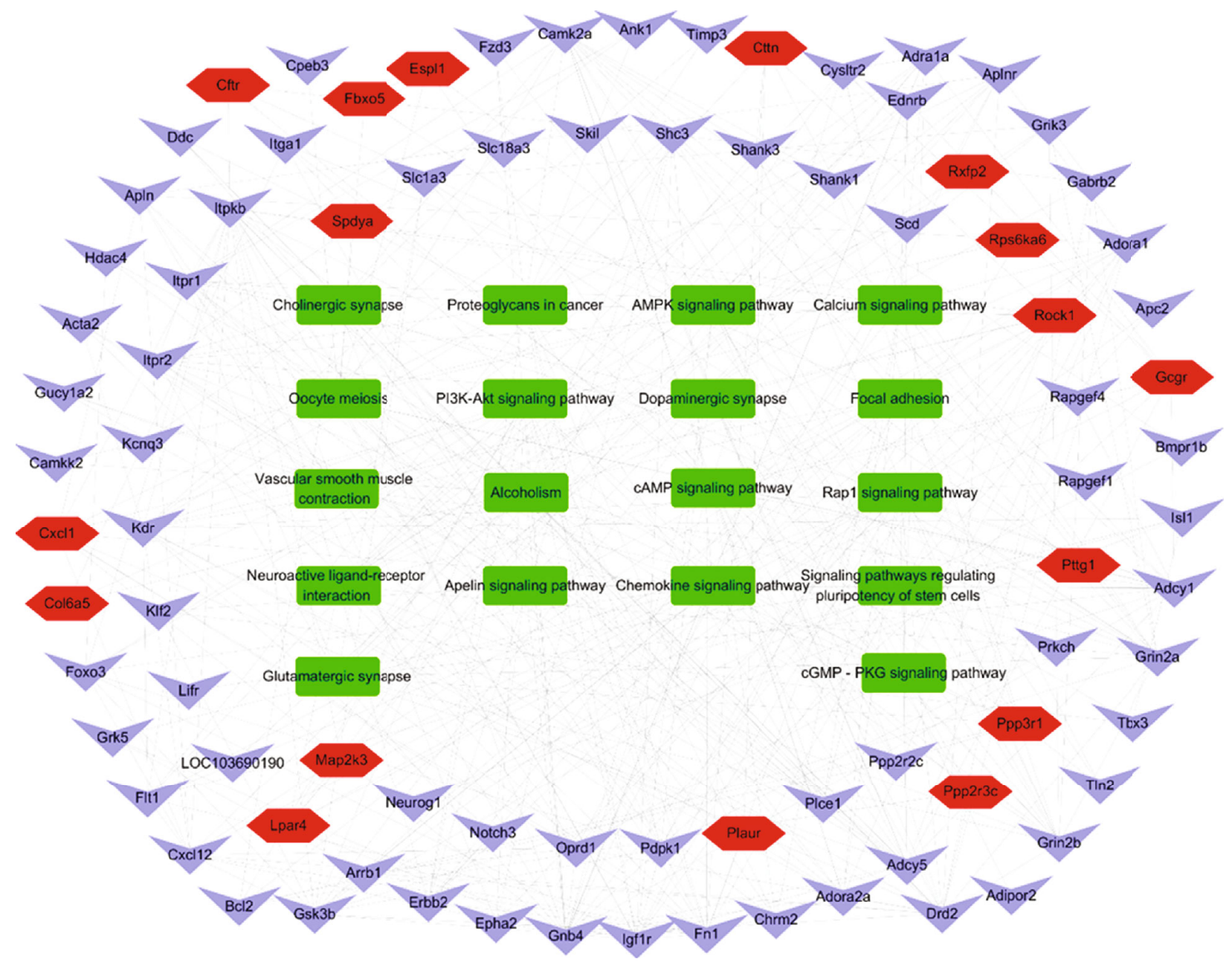

FIGURE 8: The important pathways and gene network map of PN anti-ischemic stroke. Green represents the pathways, purple represents the downregulated genes, and red represents the upregulated genes.

cerebral ischemia by protecting the NVU. The molecular mechanisms involved may include the PI3K-Akt pathway, neuroactive ligand-receptor interactions, Rap1 signaling pathway, cAMP signaling pathway, and cGMP-PKG signaling pathway. The interaction of proteins in these pathways may be potential key targets for our future understanding of the neuroprotective effects of PN in ischemic stroke.

\section{Data Availability}

The datasets and code generated or analyzed in this study are available from the corresponding author upon reasonable request.

\section{Ethical Approval}

This study was approved by the Ethics Committee of the Affiliated Hospital of Youjiang Medical University for Nationalities (Baise City, Guangxi Province, China), in accordance with the Declaration of Helsinki.

\section{Conflicts of Interest}

The authors declare that this research was conducted in the absence of any commercial or financial relationships that could be construed as potential conflicts of interest.

\section{Authors' Contributions}

Qing Huang, Lanqing Meng, Xiaohua Huang, Qiuping Chen, and Jingjie Zhao performed the main experiments. Lanqing Meng, Qing Huang, Rong Qiu, Xiaohua Huang, and Jingjie Zhao induced the animal models and collected tissues. Chongdong Jian, Qianli Tang, Jianmin Huang, Linxue Luo, and Xionglin Tang scored the disease. Xuebin Li, Yueyong Li, Jingjie Zhao, LiuZhi Wei, Qiuju Wei, Zechen Wang, and Zhongheng Wei collected data and performed data analysis. Jun Wu, Rong Qiu, Lu Huang, and Lan Li performed the transmission electron microscopy experiments. Liuzhi Wei, Qiuju Wei, Zechen Wang, Linxue Luo, Rong Qiu, Jihua Wei, Genliang Li, and Lu Huang performed RNA abstraction experiments. Yueyong Li, Xuebin Li, and Lingzhang Meng 
validated the differential expressed genes by qPCR. Lingzhang Meng and Anding Xu initiated and supervised the project and wrote the manuscript. All the authors approved the final manuscript. Lanqing Meng, Qing Huang, Xuebin Li, Ping Liang, Yueyong Li, Xiaohua Huang, Hongfei Yao, Qiuping Chen, Rong Qiu, Lan Li, Chongdong Jian and Hongfei Yao contributed equally to this study. Ping Liang substantially contributed to the design of the study especially during the initiation of this study, helped greatly establish rat model for this work, and performed part of qPCR experiments for this study. Hongfei Yao search and design most of the primers for qPCR experiments, performed functional assay through KEGG databases, and helped solve some technique problems especially when using the Internet databases.

\section{Acknowledgments}

The authors thank all the colleagues for their support and cooperations in this study. The authors would also like to thank Dr Dev Sooranna, Imperial College London, for editing the manuscript. This research was funded by grants from the National Natural Science Foundation of China (Nos. $81660791,81460614,81860226$, and 81860244), from the Guangxi Natural Science Foundation (Nos. 2019JJA140059, 2019JJA140529, 2019JJA140177, and Z2016419), from the Guangxi Clinic Medicine Research Center of Hepatobiliary Diseases (Nos. AD17129025), from the Special Funding for Guangxi Special Experts (Nos. GRCT[2019]13), and from the Guangxi Zhuang Autonomous Region Health Department Self-financing for Scientific Research Project (Z2012005).

\section{Supplementary Materials}

Supplementary Table: Longa's method of evaluation of neurological defects. The severity of neurological defects could be indicated by rat behaviors and was scored from 0 to 4 . (Supplementary Materials)

\section{References}

[1] E. J. Benjamin, P. Muntner, A. Alonso et al., "Heart disease and stroke Statistics-2019 update: a report from the American Heart Association," Circulation, vol. 139, no. 10, pp. e56-e528, 2019.

[2] W. Xie, P. Zhou, Y. Sun et al., "Protective effects and target network analysis of ginsenoside Rg1 in cerebral ischemia and reperfusion injury: a comprehensive overview of experimental studies," Cells, vol. 7, no. 12, p. 270, 2018.

[3] W. Cai, K. Zhang, P. Li et al., "Dysfunction of the neurovascular unit in ischemic stroke and neurodegenerative diseases: an aging effect," Ageing Research Reviews, vol. 34, pp. 77-87, 2017.

[4] A. Steliga, P. Kowiański, E. Czuba, M. Waśkow, J. Moryś, and G. Lietzau, "Neurovascular Unit as a Source of Ischemic Stroke Biomarkers-Limitations of Experimental Studies and Perspectives for Clinical Application," Translational stroke research, vol. 11, 2019.

[5] Y. C. Loh, C. S. Tan, Y. S. Ch'ng, C. H. Ng, Z. Q. Yeap, and M. F. Yam, "Mechanisms of action of Panax notoginseng ethanolic extract for its vasodilatory effects and partial characterization of vasoactive compounds," Hypertension Research, vol. 42, no. 2, pp. 182-194, 2019.
[6] X. Yang, X. Xiong, H. Wang, and J. Wang, "Protective effects of panax notoginseng saponins on cardiovascular diseases: a comprehensive overview of experimental studies," EvidenceBased Complementary and Alternative Medicine, vol. 2014, 13 pages, 2014.

[7] H. Zhao, Z. Han, G. Li, S. Zhang, and Y. Luo, "Therapeutic Potential and Cellular Mechanisms of Panax Notoginseng on Prevention of Aging and Cell Senescence-Associated Diseases," Aging and disease, vol. 8, no. 6, pp. 721-739, 2017.

[8] Z. Hui, D.-J. Sha, S.-L. Wang et al., "Panaxatriol saponins promotes angiogenesis and enhances cerebral perfusion after ischemic stroke in rats," BMC Complementary and Alternative Medicine, vol. 17, no. 1, p. 70, 2017.

[9] X. Shi, W. Yu, L. Liu et al., "Panax notoginseng saponins administration modulates pro- /anti-inflammatory factor expression and improves neurologic outcome following permanent MCAO in rats," Metabolic Brain Disease, vol. 32, no. 1, pp. 221-233, 2017.

[10] L. Meng, J. Lin, Q. Huang et al., "Panax notoginseng saponins attenuate oxygen-glucose deprivation/ reoxygenation-induced injury in human sh-sy5y cells by regulating the expression of inflammatory factors through miR-155," Biological \& Pharmaceutical Bulletin, vol. 42, no. 3, pp. 462-467, 2019.

[11] L. Liu, G. A. Anderson, T. G. Fernandez, and S. Doré, "Efficacy and mechanism of Panax ginseng in experimental stroke," Frontiers in Neuroscience, vol. 13, p. 294, 2019.

[12] J. Hu, C. Zeng, J. Wei et al., "The combination of Panax ginseng and Angelica sinensis alleviates ischemia brain injury by suppressing NLRP3 inflammasome activation and microglial pyroptosis," Phytomedicine, vol. 76, p. 153251, 2020.

[13] V. Rastogi, J. Santiago-Moreno, and S. Doré, "Ginseng: a promising neuroprotective strategy in stroke," Frontiers in Cellular Neuroscience, vol. 8, p. 457, 2014.

[14] L. Liu, L. Zhu, Y. Zou et al., "Panax notoginseng saponins promotes stroke recovery by influencing expression of Nogo-A, $\mathrm{NgR}$ and $\mathrm{p} 75 \mathrm{NGF}$, in vitro and in vivo," Biological \& Pharmaceutical Bulletin, vol. 37, no. 4, pp. 560-568, 2014.

[15] X. Shi, W. Yu, T. Yang et al., "Panax notoginseng saponins provide neuroprotection by regulating NgR1/RhoA/ROCK2 pathway expression, in vitro and in vivo," Journal of Ethnopharmacology, vol. 190, pp. 301-312, 2016.

[16] E. Z. Longa, P. R. Weinstein, S. Carlson, and R. Cummins, "Reversible middle cerebral artery occlusion without craniectomy in rats," Stroke, vol. 20, no. 1, pp. 84-91, 1989.

[17] S. Mohan and P. L. Foley, "Everything you need to know about satisfying IACUC protocol requirements," ILAR Journal, vol. 60 , no. 1 , pp. $50-57,2019$.

[18] A. Tsukamoto, N. Niino, M. Sakamoto, R. Ohtani, and T. Inomata, "The validity of anesthetic protocols for the surgical procedure of castration in rats," Experimental Animals, vol. 67, no. 3, pp. 329-336, 2018.

[19] I. Kwon, E. H. Kim, G. J. del Zoppo, and J. H. Heo, "Ultrastructural and temporal changes of the microvascular basement membrane and astrocyte interface following focal cerebral ischemia," Journal of Neuroscience Research, vol. 87, no. 3, pp. 668-676, 2009.

[20] H.-Q. Shi, Y. Zhang, M.-H. Cheng et al., "Sodium sulfide, a hydrogen sulfide-releasing molecule, attenuates acute cerebral ischemia in rats," CNS Neuroscience \& Therapeutics, vol. 22, no. 7, pp. 625-632, 2016. 
[21] M. Pertea, D. Kim, G. M. Pertea, J. T. Leek, and S. L. Salzberg, "Transcript-level expression analysis of RNA-seq experiments with HISAT, StringTie and Ballgown," Nature Protocols, vol. 11, no. 9, pp. 1650-1667, 2016.

[22] M. Pertea, G. M. Pertea, C. M. Antonescu, T. C. Chang, J. T. Mendell, and S. L. Salzberg, "StringTie enables improved reconstruction of a transcriptome from RNA-seq reads," Nature Biotechnology, vol. 33, no. 3, pp. 290-295, 2015.

[23] D. Kim, B. Langmead, and S. L. Salzberg, "HISAT: a fast spliced aligner with low memory requirements," Nature Methods, vol. 12, no. 4, pp. 357-360, 2015.

[24] A. Mortazavi, B. A. Williams, K. McCue, L. Schaeffer, and B. Wold, "Mapping and quantifying mammalian transcriptomes by RNA-Seq," Nature Methods, vol. 5, no. 7, pp. 621-628, 2008.

[25] M. I. Love, W. Huber, and S. Anders, "Moderated estimation of fold change and dispersion for RNA-seq data with DESeq2," Genome Biology, vol. 15, no. 12, 2014.

[26] M. D. Robinson, D. J. McCarthy, and G. K. Smyth, "edgeR: a Bioconductor package for differential expression analysis of digital gene expression data," Bioinformatics, vol. 26, no. 1, pp. 139-140, 2009.

[27] G. J. del Zoppo, "The neurovascular unit in the setting of stroke," Journal of Internal Medicine, vol. 267, no. 2, pp. 156-171, 2010.

[28] Y. Bo, Z. Jian, S. Zhi-Jun et al., "Panax notoginseng saponins alleviates advanced glycation end product-induced apoptosis by upregulating SIRT1 and antioxidant expression levels in HUVECs," Experimental and Therapeutic Medicine, vol. 20, no. 5, 2020.

[29] S. Hu, Y. Wu, B. Zhao et al., "Panax notoginseng saponins protect cerebral microvascular endothelial cells against oxygenglucose deprivation/reperfusion-induced barrier dysfunction via activation of PI3K/Akt/Nrf2 antioxidant signaling pathway," Molecules, vol. 23, no. 11, p. 2781, 2018.

[30] W. Xie, T. Zhu, X. Dong et al., "HMGB1-triggered inflammation inhibition of notoginseng leaf triterpenes against cerebral ischemia and reperfusion injury via MAPK and NF- $\kappa \mathrm{B}$ signaling pathways," Biomolecules, vol. 9, no. 10, p. 512, 2019.

[31] Q. L. Yuan, C. X. Yang, P. Xu et al., "Neuroprotective effects of ginsenoside Rb1 on transient cerebral ischemia in rats," Brain Research, vol. 1167, no. 1, pp. 1-12, 2007.

[32] X. Gao, X. Zhang, L. Cui et al., "Ginsenoside Rb1 promotes motor functional recovery and axonal regeneration in poststroke mice through cAMP/PKA/CREB signaling pathway," Brain Research Bulletin, vol. 154, pp. 51-60, 2020.

[33] D. H. D. W. Kim, D. H. D. W. Kim, B. H. Jung et al., "Ginsenoside $\mathrm{Rb} 2$ suppresses the glutamate-mediated oxidative stress and neuronal cell death in HT22 cells," Journal of Ginseng Research, vol. 43, no. 2, pp. 326-334, 2019.

[34] G. Zhang, F. Xia, Y. Zhang et al., "Ginsenoside Rd is efficacious against acute ischemic stroke by suppressing microglial proteasome-mediated inflammation," Molecular Neurobiology, vol. 53, no. 4, pp. 2529-2540, 2016.

[35] X. Huan, C. Oumei, Q. Hongmei, Y. Junxia, M. Xiaojiao, and J. Qingsong, "PDE9 inhibition promotes proliferation of neural stem cells via cGMP-PKG pathway following oxygenglucose deprivation/reoxygenation injury in vitro," Neurochemistry International, vol. 133, p. 104630, 2020.

[36] G. Carmona, S. Göttig, A. Orlandi et al., "Role of the small GTPase Rap1 for integrin activity regulation in endothelial cells and angiogenesis," Blood, vol. 113, no. 2, pp. 488-497, 2009. 